\title{
1 Sparcle: assigning transcripts to cells in multiplexed images
}

2 Authors: Sandhya Prabhakaran ${ }^{1,2}$, Tal Nawy $^{1}$ and Dana Pe'er ${ }^{11}$

$3{ }^{1}$ Computational and Systems Biology Program, Sloan Kettering Institute, Memorial Sloan

4 Kettering Cancer Center, New York, NY, 10065, USA

$5 \quad{ }^{2}$ Current address: Department of Integrated Mathematical Oncology, Moffitt Cancer Center

6 and Research Institute, Tampa, FL, 33612, USA

7 Corresponding author: peerster@gmail.com

\section{Abstract}

10 Background

11 Imaging-based spatial transcriptomics has the power to reveal patterns of single-cell gene 12 expression by detecting mRNA transcripts as individually resolved spots in multiplexed 13 images. However, molecular quantification has been severely limited by the computational 14 challenges of segmenting poorly outlined, overlapping cells, and of overcoming technical 15 noise; the majority of transcripts are routinely discarded because they fall outside the 16 segmentation boundaries. This lost information leads to less accurate gene count matrices 17 and weakens downstream analyses, such as cell type or gene program identification.

18 Results

19 Here, we present Sparcle, a probabilistic model that reassigns transcripts to cells based on 20 gene covariation patterns and incorporates spatial features such as distance to nucleus. We 21 demonstrate its utility on both multiplexed error-robust fluorescence in situ hybridization 22 (MERFISH) and single-molecule FISH (smFISH) data.

\section{Conclusions}

24 Sparcle improves transcript assignment, providing more realistic per-cell quantification of 25 each gene, better delineation of cell boundaries, and improved cluster assignments. 26 Critically, our approach does not require an accurate segmentation and is agnostic to 27 technological platform. 


\section{Keywords}

30 Spatial transcriptomics, MERFISH, osmFISH, smFISH, spatially-resolved RNA profiling,

\section{SPARCLE}

\section{Introduction}

34 Imaging-based spatial transcriptomics comprises a set of ground-breaking technologies that 35 scale up the number of genes that can be quantified within tissues (1-6). The emergence of

36 these approaches has made it possible to resolve cell type heterogeneity and to gain a 37 holistic understanding of gene expression within spatial context (7-9). However, 38 computational tools to interpret this data are severely lacking. A fundamental challenge is 39 the construction of a biologically accurate cell-by-gene count matrix, which requires cell 40 segmentation as a first step (10). Differences in cell size and morphology, occlusion, 41 overlapping cells, de-nucleated cells and technical noise from the imaging instruments make 42 simultaneous automated segmentation of multiple cell types extremely difficult.

43 The boundaries of nuclei are easier to resolve than cell outlines. Current automated 44 cell segmentation methods identify nuclei using data from a nuclear marker channel such as 45 DAPI, then radially dilate these borders by a few pixels to approximate cell boundaries. This 46 tends to leave a substantial fraction of 'dangling' transcripts outside of defined cell 47 boundaries (Fig. 1). Dangling transcripts are potentially assignable to multiple cells, 48 especially as distance from the nucleus increases, and are thus discarded prior to 49 generating the count matrix. Moreover, 2D images only reveal a slice of the cell volume. The transcripts present in peri-nuclear sections therefore represent a very sparse sampling of the 51 cellular transcriptome, which underpowers downstream analyses of cell types and gene 52 programs.

To address these issues, we introduce Sparcle (spatial reassignment of spots to cells 54 via maximum likelihood estimation), a comprehensive probabilistic model that assigns 
55 dangling transcripts on the basis of gene covariance, as well as the transcripts' distance

56 between neighboring cells and adjacent transcripts. These patterns include neighboring cells

57 (defined by Euclidean distances between cell centroids), and neighboring transcripts for

58 each cell. Sparcle generalizes to any smFISH imaging methodology, and we demonstrate its

59 utility on both multiplexed error-robust fluorescence in situ hybridization (MERFISH) (9) and

60 single-molecule FISH ( $\mathrm{smFISH})(5,8)$ data. Sparcle is one of the first methods to capture

61 dangling transcripts; we show that it recovers more than $50 \%$ of these mRNAs, and validate

62 its performance using scRNA-seq data.

\section{Results}

\section{Sparcle model}

66 Sparcle is a probabilistic model that iteratively assigns dangling transcripts to neighboring

67 cells using maximum likelihood estimation (Methods). Importantly, our approach does not require a highly accurate segmentation as input.

69 The large number of transcripts in each cell justifies modelling gene expression as a 70 multivariate Gaussian distribution, based on the central limit theorem assumption. An image 71 can thus be considered as a mixture of cell-specific Gaussian distributions, which can be 72 posed in a Dirichlet process mixture model (DPMM) (11-13) setting to identify 73 heterogeneous cell types. In Sparcle, the user can choose either the DPMM or Phenograph 74 (14) for clustering; neither require that cluster numbers are known a priori. We then compute

75 a multivariate Gaussian distribution representing gene-gene covariation relationships for 76 each cluster in our data by learning the cluster-specific first and second order moments 77 (mean and covariance) of each cluster. For every dangling mRNA, the algorithm constructs 78 a circular weighted 'mock' cell with radius $r$, centered at the mRNA, that captures proximal 79 mRNA neighbors (Fig. S1 and Methods). The contribution of each gene to the mock cell is 80 inversely weighted by its distance from the dangling mRNA. Next, Sparcle generates a 81 maximum likelihood estimate (MLE) of which cluster (often corresponding to cell type) the 
82 mock cell most likely represents and assigns the dangling mRNA to the cell with that cluster

83 label nearest to the mock cell, and updates the count matrix. This process of assigning

84 dangling mRNAs continues for a fixed number of iterations by updating and clustering the

85 count matrix, recalculating the cluster-specific moments and revising the cellular

86 assignments to clusters at each iteration. The number of robustly assigned transcripts per

87 iteration exhibits asymptotic behavior, and we have found little additional benefit beyond the

88 default of 3 iterations. With a user-provided cell segmentation and a minimal set of default

89 parameters, Sparcle can robustly assign dangling mRNAs in under $10 \mathrm{~min}$ on a high-

90 performance cluster for a typical MERFISH FOV consisting of $\sim 80$ cells and $\sim 14,000$

91 dangling mRNAs.

92 The outputs of the iterative model are a) a more realistic count matrix, based on

93 improved mRNA assignments to cells, b) cluster assignments for each cell and c) improved

94 delineation of cell boundaries.

96 Evaluating Sparcle using matching scRNA-seq data

97 The mammalian brain is characterized by diverse cell types with complicated morphologies

98 and cellular interactions that can greatly benefit from multiplexed imaging and spatial 99 analysis. A MERFISH image of the mouse hypothalamus posterior optic region comprises $100 \sim 30,000$ cells from 70 different neuronal populations and 140 assayed genes (9) (Fig. 1). 101 From this image, 400 smaller fields of view (FOVs) were imaged at high magnification (Fig. 102 1a); segmentation using dilated cell nuclei revealed that $\sim 52 \%$ of mRNAs, which exhibit 103 substantial spatial heterogeneity, remain unassigned (Fig. 1b,c). Using these individual 104 FOVs (Fig. 1d-f), Sparcle increases the fraction of total transcripts that are assigned to cells 105 by assigning 24.2, 8.6 and $2.3 \%$ out the total transcripts in iterations 1,2 and 3 , respectively 106 (Fig. 1g), ultimately capturing $68 \%$ of the 2.8 million dangling transcripts in this full dataset. To evaluate the cell populations identified by Sparcle, we used paired scRNA-seq 108 data (31,299 cells) as ground truth, considering a subset of 900 genes containing MERFISH 109 probes and neuronal genes, as utilized in Moffitt et al. [9], to call cell types. We used 
110 Phenograph (14) to cluster both the scRNA-seq and MERFISH count matrices before and

111 after applying Sparcle. Post-Sparcle, we observe that the cell type proportions are more 112 concordant with those of the scRNA-seq data (Fig. 2a).

113 To further assess Sparcle's performance, we tested the similarity of count matrices 114 derived from MERFISH and scRNA-seq using two different covariance approaches 115 (Methods). The Gramian (cluster-cluster) covariance matrices from scRNA-seq and 116 MERFISH are more similar after Sparcle $(p=0.620$, Box's M test; Frobenius norm $=2.34)$ 117 than before Sparcle $(p=0.309$; Frobenius norm $=15.18$; larger $p$-values and smaller norms 118 indicate more similar matrices), showing that Sparcle assignments improve MERFISH cell 119 type calls relative to ground truth (Fig. $\mathbf{2 b}$ ). The cluster-cluster cross covariance matrix 120 between scRNA-seq and post-Sparcle MERFISH exhibits stronger variances within clusters 121 (dominant diagonal) than between clusters, compared with the pre-Sparcle cross covariance 122 matrix, especially for non-neuronal cell types (Fig. 2c). This covariance pattern signals 123 stronger relationships within cell types and weaker relationships between different cell types, supporting the accuracy of Sparcle assignments.

125 Finally, Sparcle clearly improves the assignment of canonical cell-type-specific genes 126 for most clusters, including excitatory neuronal, endothelial, ependymal and astrocyte cells 127 (Fig. 2d). This observation supports the validity of the higher proportions of these cell types 128 that result from Sparcle transcript assignments (Fig. 2a).

130 Sparcle captures more information in MERFISH-derived cell clusters

131 We sought to determine whether Sparcle assignments capture more transcriptional 132 variability and lend greater structure to molecular quantification data from imaging datasets. 133 To do this, we examined pairwise Pearson correlation coefficients between cluster 134 expression profiles extracted from the mouse hypothalamus scRNA-seq and MERFISH data, 135 both before and after Sparcle, and used Loewner ordering to compare matrices (see 136 Methods). 
137 We first extracted and examined two broad neuronal cell types. We built a pre-

138 Sparcle MERFISH matrix with 100 randomly sampled excitatory cells stacked over 100 139 inhibitory cells and a post-Sparcle matrix with the same cells, then computed Pearson 140 correlations with a similarly constructed scRNA-seq matrix of 100 random cells per cell type

141 (Fig. 3a). The matrices reveal higher scRNA-seq correlations and greater correlation 142 structure for both cell types after using Sparcle (Fig. 3a). Notably, Sparcle assignments 143 substantially improve the recovery of excitatory cells, which only number approximately half 144 of the inhibitory cells. Furthermore, Loewner ordering reveals that the excitatory block in the 145 post-Sparcle correlation matrix captures additional variability (subtracting this block in the 146 post-Sparcle matrix from the pre-Sparcle matrix generates a positive semi-definite matrix, 147 whereas subtraction in the other direction does not).

$148 \quad$ For non-neuronal clusters, we placed astrocytes, endothelial and ependymal cells in 149 one set (NN set 1) and immature and mature oligodendrocytes, microglia and mural cells in 150 another (NN set 2), and performed Pearson correlation and Loewner ordering (Fig. 3b). Both 151 cell sets show clearly higher correlations with scRNA-seq, and the Pearson correlation block 152 diagonals in the post-Sparcle matrix indicate stronger relationships. Loewner ordering of the 153 individual blocks likewise indicates that dangling transcript assignment captures more 154 variability post-Sparcle. This additional variability can be exploited to refine cell type 155 assignment and improve gene-level analyses.

157 Sparcle generalizes to additional imaging modalities

158 To test the generalizability of Sparcle, we examined public single molecule FISH (smFISH) 159 images of the adult mouse primary visual cortex, comprising 3500 cells and $1,074,000$ 160 mRNA spots from 22 unique genes (15) from which we subsampled 250,000 mRNAs 161 (154,000 within cells (61.8\%) and 95,000 dangling (38.2\%)). For comparison purposes, we 162 used matching scRNA-seq data comprising 43,498 cells and 727 highly variable genes, 163 including the $22 \mathrm{smFISH}$ genes. We clustered count matrices and annotated smFISH cells 164 and clusters (both before and after Sparcle) based on the four cell types identified by 
165 scRNA-seq (Fig. 4a and Fig. S3). Sparcle recovers $32.2 \%$ of the total mRNA across three 166 iterations (26.3\% in iteration $1,4.7 \%$ in iteration 2 and $1.2 \%$ in iteration 3 ) (Fig. S4). Analysis 167 of Gramian matrices reveals that post-Sparcle cell-type covariances are more similar to the matching scRNA-seq cluster covariances (Box's M test, $p>0.05$; Frobenius norm closer to

0) (Fig. 4b) and the cluster-cluster cross covariance matrix between scRNA-seq and

170 MERFISH has a stronger diagonal matrix post-Sparcle (Fig. 4c). Cluster analysis shows that

171 Sparcle assigns multiple genes to at least one additional biologically relevant cell type; for 172 example, Kcnk2, Sv2C and Thsd7a belong to either endothelial or excitatory cells pre173 Sparcle, and expand to both cell types after Sparcle (Fig. 4d).

\section{Discussion}

176 Sparcle addresses an important need in the rapidly evolving field of single-cell spatial 177 transcriptomic imaging - the recovery of transcripts outside of segmentation cell boundaries.

178 Our approach assigns dangling transcripts to cells based on their gene expression similarity, through an imaging-platform-independent and comprehensive probabilistic model. no ground truth for cell segmentation, and many cell morphologies are very complex; for example, neuronal cell bodies are typically partially segmented, whereas mRNA-bearing axons are not usually segmented at all. On MERFISH and smFISH images of brain sections, Sparcle recovers more than $50 \%$ of dangling mRNAs, driving improved cell type calls and gene-level analyses. We have shown that Sparcle mRNA assignments lead to refined count matrices and improved assessment of cell type heterogeneity. (SSAM) (16) and Bayesian Segmentation of Spatial Transcriptomics Data (Baysor) (17) are recent quantification approaches that aim to circumvent the need for cell segmentation. Like Sparcle, Baysor works by assigning transcripts to artificially generated mock cells. However,

191 Baysor requires that a minimum number of transcripts per cell be specified a priori. The 192 approach assumes that cells consist of homogenous transcripts (whereas cells and their 
193 neighbors can exhibit a diverse mix of mRNAs, e.g. Fig. 1c), that they have a fixed number

194 of mRNA neighbors (whereas Sparcle allows neighborhood counts to vary within a fixed 195 neighborhood size), and that cells take on an ellipsoid shape through a bivariate normal prior 196 (not the case for many neuronal cells, for example).

197 Sparcle relies on imperfect region-independent cell segmentations as a starting point,

198 and it uses MLE to assign transcripts to inferred cell clusters. Given the high number of 199 dangling mRNAs, this a relatively simple, computationally cheap and powerful strategy that 200 does not require customization for different imaging protocols. In contrast, Baysor requires 201 that its core EM machinery be tailored to each imaging protocol.

202 A crucial assumption for SSAM is the uniform distribution of mRNA within a cell, 203 which is unrealistic given the diversity of mRNA within a cell (Fig. 1c and Fig. S1). SSAM 204 further requires the approximation of an average cell diameter, which must be adjusted for 205 every imaging scenario. This also means that the width of the Gaussian kernel used in the 206 KDE needs to be chosen based on the imaging protocol. A wrong choice of bandwidth can 207 lead to signal contamination from adjacent cells.

208 Transcript assignment by Sparcle can be further improved. Sparcle currently utilizes an MLE protocol which does not assume an informative prior distribution for the dangling 210 mRNAs. For any given gene, we can introduce a prior distribution for the localization of its 211 transcripts and implement a maximum a posteriori (MAP) Bayesian inference procedure 212 based on their spatial diversity. Additional improvements can be achieved by staining for 213 cellular membranes in addition to nuclei, to provide rough boundaries for entire cells. This 214 improved segmentation would help to resolve dangling transcripts positioned farther from the 215 nucleus. A software version in a language such as Java may also run more efficiently.

216 Sparcle is shown to effectively assign dangling mRNAs to cells and correct the count 217 matrices. We hope that the computational infrastructure provided by Sparcle will further 218 contribute to the emerging landscape of high-throughput spatial transcriptomic image 219 analysis. 
bioRxiv preprint doi: https://doi.org/10.1101/2021.02.13.431099; this version posted February 14, 2021. The copyright holder for this preprint (which was not certified by peer review) is the author/funder, who has granted bioRxiv a license to display the preprint in perpetuity. It is made available under aCC-BY 4.0 International license. 


\section{Methods}

\section{Algorithmic overview}

225 As input, Sparcle takes approximate cell segmentations generated using any method for an 226 FoV. The cell segments across all the FoVs are collected, and Sparcle creates a global 227 count matrix of cells and genes, which is clustered to identify cell types. The large number of 228 transcripts in each cell justifies modelling gene expression as a Gaussian distribution, based 229 on the central limit theorem assumption. An image can thus be considered as a mixture of 230 cell-specific Gaussian distributions, which can be used in a Dirichlet process mixture model 231 (DPMM) for identifying heterogeneous cell types. First, Sparcle learns the cluster-specific 232 first and second order moments (mean and covariance) of each cluster. Next, for each 233 dangling mRNA, a mock cell is created using mRNA transcripts occurring within a certain 234 radius of the dangling mRNA. The mock cell is compared to previously computed cluster235 specific moments with the objective of maximizing the likelihood of a dangling mRNA being similar to a cluster and then assigning the mRNA to the closest neighboring cell belonging to

237 the most similar cluster. Neighboring cells are calculated based on Euclidean distances 238 between the mRNA and cell centroids. As each mRNA gets assigned to a cell, we update 239 the count matrix. At the onset of each subsequent iteration, the updated global count matrix 240 is re-clustered, the cluster-specific moments are recomputed and the assignment of dangling 241 mRNAs continues. Through this construction, we are, in essence, building a global model 242 with global cluster-specific means and covariances that all the FoVs have contributed to and 243 will therefore adhere to. Using this global approach, we can ensure that the underlying 244 probabilistic model is constant across FoVs per iteration as well as across iterations.

245 If we cluster each FoV separately in order to build a local structure, it becomes necessary to 'match' the clusters between FoVs. This can be both computationally intensive

247 and unstable, because not all cell types are present in all FoVs, and thus, clusters can differ 248 across FoVs. To build the global structure, we need to perform roughly $400 \times \mathrm{k} \times \mathrm{k}$ ' pairwise 249 stable matching of clusters between FoVs, where k and k' are the number of clusters for any 
250 given pair of FoVs. These comparisons would be riddled by missing or unseen clusters,

251 leading to an improper global structure.

252 Sparcle therefore resorts to constructing a global structure of cell types from the 253 beginning, to ensure a reliable set of clusters to which dangling mRNAs can be assigned.

254 Sparcle comprises several canonical building blocks, including clustering to identify cluster-

255 specific moments, constructing a mock cell for each dangling mRNA to enable comparison

256 of a dangling mRNA to cluster moments, and maximum likelihood estimation.

Mockcell window size and shape choice

259 The mockcell is designed as a circle centered at the dangling mRNA. We consider a circular 260 window to approximate the cell body of an average neuronal cell. To estimate the 261 approximate radii of two mockcells $A$ and $B$, we draw bounding boxes around those cells 262 (see Fig. S2 for example corresponding to calculations below). The area of a cell's bounding box $=\left(x_{1}-x_{2}\right) *\left(y_{1}-y_{2}\right)$. We equate this to the area of a circle $=\pi r^{2}$, to estimate $r$.

Area of Cell $A=(636-567) *(1200-1113)=\pi r^{2}$

266

267

Area of Cell $\mathrm{B}=(1548-1404) *(1569-1461)=\pi r^{2}$

$$
\rightarrow r=70 \text { pixels }
$$

271 Since most cells in MERFISH and smFISH data have $r<=75$ pixels, we have set $r=80$

272 pixels as the default radius for a mockcell.

\section{Weighted mockcell creation}

275 Every dangling mRNA is represented as a mockcell and is a vector of length equal to the 276 number of genes, G. Using the circular window of radius $r$ centered at the dangling mRNA, a 277 mockcell is constructed using an inverse distance weighting (IDW) method where distances 278 from the dangling mRNA (or the center) to each of the neighboring mRNA falling within $r$ are 279 interpolated to create a weighted average for each $\mathrm{g}$ in G. With this construction, mRNAs 
280 closest to the dangling mRNA have more influence than those farther away. The weighted

281 average $u_{g}$ for each gene $g$ for the mockcell centered at $x$ is calculated based on the

282 simplest weighting function or Shepard's method (18) that uses the weights in inverse power

283 as:

$284 \quad u_{g}(x)=\left(\frac{\sum_{i=1}^{N} w_{i}(x) u_{i}}{\sum_{i=1}^{N} w_{i}(x)}\right)^{2}$ when $\mathrm{d}\left(\mathrm{x}, \mathrm{x}_{\mathrm{i}}\right) !=0$

285 and

$286 w_{i}(x)=\left(\frac{1}{d\left(x, x_{i}\right)}\right)^{2}$ where $\mathrm{d}\left(\mathrm{x}, \mathrm{x}_{\mathrm{I}}\right)$ is the distance between the interest point $x$ and a point in the 287 neighborhood $x_{i}$ (denoting a neighboring $\mathrm{mRNA}$ ) where there are $i=1, \ldots N$ observations of 288 gene $g$ in the neighborhood and $w_{i}(x)$ is the weight or predicted value at point $x$.

289 We use a modification of Shepard's method that calculates the interpolated value 290 using only nearest neighbors within the r-sphere (instead of the entire sample space). 291 Weights in the above equation are modified in this case to give $w_{i(\text { modified })}(x)=$ $292\left(\frac{\max \left(0, \mathrm{r}-\mathrm{d}\left(x, x_{i}\right)\right)}{\mathrm{r} * \mathrm{~d}\left(x, x_{i}\right)}\right)^{2}$

293 that are used to compute the modified weighted average $u_{g_{-} \text {mod }}(x)$ which is entered for each $294 g$ the mRNA accounts for in the mockcell.

296 Spatial distances and how they are incorporated into Sparcle

297 By orienting every image to a 2D Cartesian coordinate system, we assume each image to 298 reside on a Euclidean plane. This allows us to calculate the inter-cellular distances using 299 pairwise Euclidean distances between cell centroids. Further, the local concentration of 300 mRNA transcripts within a mockcell is derived by computing the Euclidean distances 301 between the transcripts and the mockcell's centroid.

303 Maximum likelihood method

304 We assume that the gene expression in each cell follows a multivariate Gaussian 305 distribution. This enables us to view an image as a mixture of cell-specific Gaussian 
306 distributions, and to use a Dirichlet process mixture model (DPMM) for clustering the cells

307 into $K$ different cell types.

308

In order to assign a dangling mRNA, $m$, to one of the inferred cell types $k=1 \ldots K$,

309 we use the maximum likelihood (ML) method. Assuming there are $k$ cell types, the mockcell

310 originating at a dangling mRNA, is tested for each cell type's parameters i.e. multivariate

311 mean $\mu_{k}$ and covariance $\Sigma_{k}$ to identify $k_{M L}$ that maximizes the likelihood distribution. We

312 assume the likelihood distribution to be that of a multivariate Gaussian. The analytic form of

313 the ML method can be written as:

$$
\begin{gathered}
\hat{\theta}_{\mathrm{ML}}=\arg \max \quad \quad \theta \in \Theta p\left(m \mid \mu_{k}, \Sigma_{k}\right) \text { and } \\
p\left(m \mid \mu_{k}, \Sigma_{k}\right)=\frac{\exp \left(-0.5\left(\mathrm{~m}-\mu_{\mathrm{k}}\right)^{\mathrm{T}} \Sigma_{\mathrm{k}}^{-1}\left(\mathrm{~m}-\mu_{\mathrm{k}}\right)\right)}{\sqrt{(2 \pi)^{\mathrm{d}}-\left|\Sigma_{\mathrm{k}}\right|}}
\end{gathered}
$$

315 where $\hat{\theta}_{\mathrm{ML}}$ is the maximum likelihood estimate of the Gaussian parameters $\theta=\left[\mu_{k}, \Sigma_{k}\right], \Theta$ is

316 the finite-dimensional parameter space for $\theta, d$ is the number of genes and $p(m \mid \theta)$ is the

317 non-degenerate case of the multivariate Gaussian probability density function (pdf). We

318 ensure the non-degenerate pdf by constructing positive (semi) definite $\Sigma_{k}$ using Givens

319 rotations as described in the next section. The dangling mRNA is assigned to the nearest

320 neighboring cell belonging to the closest 'likely' cell type, $k_{M L}$ described by $\hat{\theta}_{\mathrm{ML}}$. The count

321 matrix entry for the cell identified and gene associated by the dangling mRNA is updated.

322 The assigned dangling mRNA is removed from the candidate list of mRNAs to be assigned.

323 The ML method is known to be optimal for large data sizes (19).

\section{Givens rotations on the covariance matrices}

326 To ensure that covariance matrices used in the maximum likelihood density estimation are

327 positive semi-definite, and to avoid unnecessary cost overhead by inverting these matrices, 328 we perform QR decomposition using the Givens rotations on the covariance matrices. These 329 are efficient transformations on sparse matrices and can be better parallelized as well. 
330 For any real square matrix $A$, the $Q R$ decomposition is $A=Q R$ where $Q$ is an

331 orthogonal matrix and $R$ is an upper triangular matrix. QR decompositions are computed

332 with a series of Givens rotations. In each rotation an element in the subdiagonal of the matrix

333 is zeroed out, to form the symmetric R matrix. The product of all the Givens rotations forms

334 the orthogonal Q matrix.

335 In practice, Givens rotations are not actually carried out by building the entire matrix

336 to perform the matrix multiplication between $Q$ and $R$. Instead, only the upper triangle of $R$ is

337 built out and multiplied with $Q$ during each Givens rotation which is equivalent to a sparse

338 Givens matrix multiplication, without having to explicitly handle the sparse elements. This

339 Givens rotation procedure is useful when only a relatively few off diagonal elements need to

340 be zeroed out implying the matrix is still dense, and can be more easily parallelized $(20,21)$.

342 Runtime complexity analysis

343 We calculate the Big O notation for an entire run of Sparcle for one MERFISH FOV. On 344 average, a MERFISH FOV consists of 80 cells, of which $\sim 50 \%$ of mRNA transcripts fall 345 within a cell and the rest are dangling mRNA. We consider $\mathrm{n}$ the number of cells, $d$ the 346 number of genes, $m$ the number of dangling mRNA transcripts in the first iteration, $m^{\prime}$ the 347 number of dangling mRNA transcripts in the subsequent iterations, $x$ the number of line 348 segments constituting each cell boundary, $k$ the number of mRNA neighbors per dangling 349 mRNA for constructing the mockcell and $c$ the total number of iterations. We have observed 350 that setting $c$ to 3 is usually sufficient since most of the dangling mRNAs are assigned in 351 iterations 1 and 2.

1. Construct the count matrix for each FOV and consolidate to an overall count matrix: $O(n), 1 \mathrm{~min}$

2. Iteration $1: \mathrm{O}\left(n d^{2}\right)+\mathrm{nO}(\mathrm{n})+\mathrm{mO}(\mathrm{k})=\mathrm{O}\left(n d^{2}\right), 3$ mins

a. Maximum likelihood estimation calculations: $O\left(n d^{2}\right)$

b. Euclidean distance to find neighboring cells for cell $i: \mathrm{O}(\mathrm{n})$

i. n subtractions for (cell_centroid_i - neigh_cell_centroid)

ii. $n$ squares of (i) 
iv. Final one square root. (So each of these is (at most) linear in n, and hence so is the whole algorithm.)

c. Perform $b$ for $\mathrm{n}$ cells $=\mathrm{nO}(\mathrm{n})$

d. Euclidean distance to create $\mathrm{j}^{\text {th }}$ mock cell using neighboring mRNA for $\mathrm{j}^{\text {th }}$ dangling mRNA: $\mathrm{O}(\mathrm{k})$

i. $\quad k$ subtractions for (dangling mRNA_centroid_j - neigh_mRNA)

ii. $\quad k$ squares of (i)

iii. $\quad k-1$ further additions to add (ii)

iv. Final one square root. (So each of these is (at most) linear in $k$, and hence so is the whole algorithm.)

e. Perform $d$ for $m$ cells $=m o(k)$
4. Final Iteration (combining all the assignments and plotting): $O(n m)+c O(m), 1.5$ mins

3. Iteration $c: 0\left(n d^{2}\right), 2.5$ mins

Therefore, overall runtime complexity is $\mathrm{O}(\mathrm{n})+\mathrm{O}\left(n d^{2}\right)+\mathrm{cO}\left(n d^{2}\right)+\mathrm{O}(\mathrm{nm})+\mathrm{cO}(\mathrm{m}) \sim$ $\mathrm{O}\left(\mathrm{nd}^{2}\right)+\mathrm{O}(\mathrm{nm}) \sim O(n m) \sim O(m)$

The runtime is given based on Sparcle runs on an HPC.

\section{Evaluating cell populations identified by Sparcle using matching scRNA-seq}

(a) The Gramian matrix across clusters for the pre-Sparcle count matrix, post-Sparcle count matrix and matching single-cell RNA-seq data is shown in Fig. 2b. For this, we compute the Box's $M$ test which is a parametric test to check the homogeneity of variance (homoscedasticity). Specifically, it tests for the homogeneity of covariance matrices describing multivariate Gaussian data according to one or more groups. The groups in our setting are pre-Sparcle, post-Sparcle and scRNA-seq. The test compares the product of the log determinants of each of the covariance matrices to the log determinant of the pooled covariance matrix, similar to a likelihood ratio test (22). The generated test statistic is called Box's M statistic and is approximated using a chi-square goodness of fit. The null hypothesis for this test is that the observed covariance matrices are equal across groups. This means a non-significant test result (i.e. one with a large p-value) indicates that the covariance matrices are not different $(23,24)$. 
The Box's M test between

- Pre-Sparcle and scRNA-seq: median chi-squared $=0.2556, \mathrm{df}=2, \mathrm{p}$-value $=0.3091$.

- Post-Sparcle and scRNA-seq: median chi-squared $=0.9458, \mathrm{df}=2, \mathrm{p}$-value $=0.6201$.

A p-value greater than 0.05 indicates the variances are homogeneous and higher $p$ values indicate more homogeneous variances depicting that post-Sparcle and scRNAseq covariances are more similar. Further, we check the distance between

401

(b) The Cluster-cluster cross covariance matrix between pre-Sparcle clusters with scRNAseq clusters and between post-Sparcle clusters with scRNA-seq clusters (Right panel) is shown in Fig. 2c.

406 We observe that in the post-Sparcle covariance,

407 a. the diagonal shows stronger variances within clusters than the pre-Sparcle covariance matrix, especially for nonneuronal cell types. opposed to the pre-Sparcle and scRNA-seq covariance.

412 This is a preferred covariance pattern that signals stronger relationships within and 413 weaker relationships between cell types. This means that cell types have garnered 414 relevant mRNA transcripts via Sparcle assignments.

416 (c) We map canonical genes per cluster for both pre-Sparcle and post-Sparcle cell types in 417 Fig. 2d. We see clear and improved cluster assignments for most of the post-Sparcle $418 \quad$ clusters. 
421 Consider a $\mathrm{m} \times \mathrm{m}$ covariance matrix $A$ and the set $B$ with all $\mathrm{m} \times \mathrm{m}$ positive definite 422 covariance matrices having similar structure (for example, uniform covariance structure). In 423 order to find the discrepancy between $A$ and the set $B$, we define $\mathrm{D}(\mathrm{A}, \mathrm{B})=\min _{\left\{\mathrm{B}^{\mathrm{B}} \mathrm{in} \mathrm{B}\right\}} L(A, B)$ 424 where $L(A, B)$ is a measure of the distance between the two $\mathrm{m} \times \mathrm{m}$ matrices $A$ and $B$. We 425 consider $A$ the covariance matrix from the scRNA-seq measurements and the set $B$ to 426 consist of the pre- and post-Sparcle covariance matrices that originate from the same 427 measurement space. The matrix $B^{*}$ which has the smallest discrepancy can be viewed as 428 that with the most likely or closest to the underlying structure of $A$. We consider the distance 429 between the two matrices to be the square of the Frobenius-norm, or the F-norm: $L(A, B)=$ $430 \operatorname{tr}\left((A-B)^{T}(A-B)\right)$, which is defined as the square of the sum of the absolute squares of 431 its elements (25).

433 For Fig. 2b, the Frobenius norm for the Gramian matrices between

- Pre-Sparcle and scRNA-seq is 15.18

- Post-Sparcle and scRNA-seq is 2.34 showing that the post-Sparcle covariance is closer to the scRNA-seq covariance.

Loewner ordering of matrices

439 One way to compare matrices is by using the positive definiteness (pd) or positive-semi 440 definiteness (psd) property. We extract the block matrices from the Pearson's correlation 441 matrix (Fig. 3) and test them for pd or psd using the Cholesky decomposition. Cholesky 442 decomposition factors a psd matrix $A$ into: $A=L L^{T}$ where $L$ is the lower triangular matrix 443 and also the Cholesky factor of $A$. If $\mathrm{L}$ is invertible, then $\mathrm{A}$ is a pd matrix. Since the blocks 444 are from covariance matrices, the blocks satisfy either the pd or psd property. Assume there 445 are two covariance matrices (or multivariate variances) $C$ and $D$ where $C$ and $D$ are by 446 definition pd or psd or verified using the Cholesky decomposition described above. If upon 447 removing $D$ from $C$ (i.e. $C-D$ ) we get a positive (semi) definite matrix that means that $C$ has 
448 captured more viable variability within the system. We use this Loewner ordering of

449 covariance matrices to show that the post-Sparcle covariance matrices capture additional

450 real variability which went unaccounted for in the pre-Sparcle covariance matrices.

\section{Declarations}

452

453 Ethics approval and consent to participate

454 Not applicable

455

456 Consent for publication

457 Not applicable

458

\section{Availability of data and materials}

460 MERFISH data and corresponding scRNA-seq data can be found at (9). For MERFISH, 461 there are 400 FOVs, each $~ 3 G B$ and 4.1 Megapixels in size. There are 31,241 cells across 462 the 400 images of which 2.7 million mRNA transcripts fall within cells and 2.8 million are 463 dangling mRNA. The matching scRNA-seq data has 31,299 cells and 27,998 genes.

464 For smFISH, we utilise a single image that was stitched and provided by Brian Long from the 465 Allen Brain Institute. The image is that of the primary visual cortex (VISp) region of an adult 466 mouse brain. There are 3500 cells and 22 genes. There were 1074,000 mRNA within this 467 image of which we subsample 250,000 mRNA of which 154,000 mRNA are within cells and 46895,000 are dangling mRNA that is used by Sparcle. The matching scRNA-seq data had 46943498 cells and 45,000 genes and is available at (15).

470 The open-source, platform independent software implementation of Sparcle is available on 471 Github: https://github.com/sandhya212/Sparcle for spot reassignments

472 Code is written in Python 3 and is parallelized to process multiple FOVs during each 473 iteration. We have also developed code that is necessary to reconstruct each FOV from the 474 stage coordinates to image coordinates, plot the cell segment boundaries obtained from the 475 segmentation, and plot the mRNA within cells and dangling mRNAs. 
477 Competing interests

478 The authors declare that they have no competing interests.

479

$480 \quad$ Funding

481 This work was supported by NIH grants U54-CA209975, U2C-CA233284, DP1-HD084071

482 (DP) and Cancer Center Support Grant P30-CA008748.

483

484 Author's contributions

485 SP, DP conceived the study and designed the concept and idea of SPARCLE. SP 486 developed the code for SPARCLE. SP acquired and performed data analyses on MERFISH 487 and smFISH data. TN provided critical comments and discussions. DP supervised the study. 488 All authors wrote and approved the final manuscript.

489

490 Acknowledgements

491 We thank Jeffrey Moffitt (Harvard University) for sharing MERFISH data and for in-depth 492 discussions on biological interpretation of the data. We also thank Brian Long and Jeremy 493 Miller for sharing the Allen smFISH data and thoughts on the matching single-cell RNA-seq 494 data. 


\section{$497 \quad$ Figures}

498 Figure 1: Sparcle captures transcriptomic diversity that is present in spatial transcriptomic 499 images but is discarded by typical analysis. a-f. A single 2D exemplar field of view (FOV) of 500 the mouse hypothalamic posterior optic region imaged by multiplexed error-robust 501 fluorescence in situ hybridization (MERFISH) [9]. a. MERFISH image showing individual 502 transcripts as points of green fluorescence. b. Conservative segmentation captures $n=$ 50312,000 transcripts (blue dots; $48 \%$ of mRNAs) (Moffitt et al., Science 2018), leaving $504 \sim 14,000$ 'dangling' transcripts (pink crosses). Current methods fail to accurately segment 505 neuronal cell morphologies in particular, which often contain transcripts far from the cell 506 body. c. Spatial diversity of transcripts within (dots) and outside (crosses) the cell 507 segmentation. Each mRNA is colored according to corresponding gene ( $\mathrm{n}=140$ genes 508 assayed). Inset, magnification highlighting the diversity of dangling transcripts; solid lines 509 indicate segmentation boundaries. d-f. For the FoV shown in (a), Sparcle captures 27\%, $51010.3 \%$ and $1.2 \%$ of total mRNAs in iteration 1 (d), iteration 2 (e) and iteration 3 (f), 511 respectively. (Panel (f) also overlays all the mRNA assignments from previous iterations). $\mathbf{f}$.

512 Overall dangling mRNA assignments across the three iterations, with unassigned dangling 513 mRNAs removed. Dangling mRNAs assigned to one cluster are connected to the cell 514 centroid by lines of the cluster color; black dots are unassigned dangling mRNAs. g. Fraction 515 of all mRNAs recovered across 400 MERFISH FOVs by segmentation and Sparcle 516 assignment. 

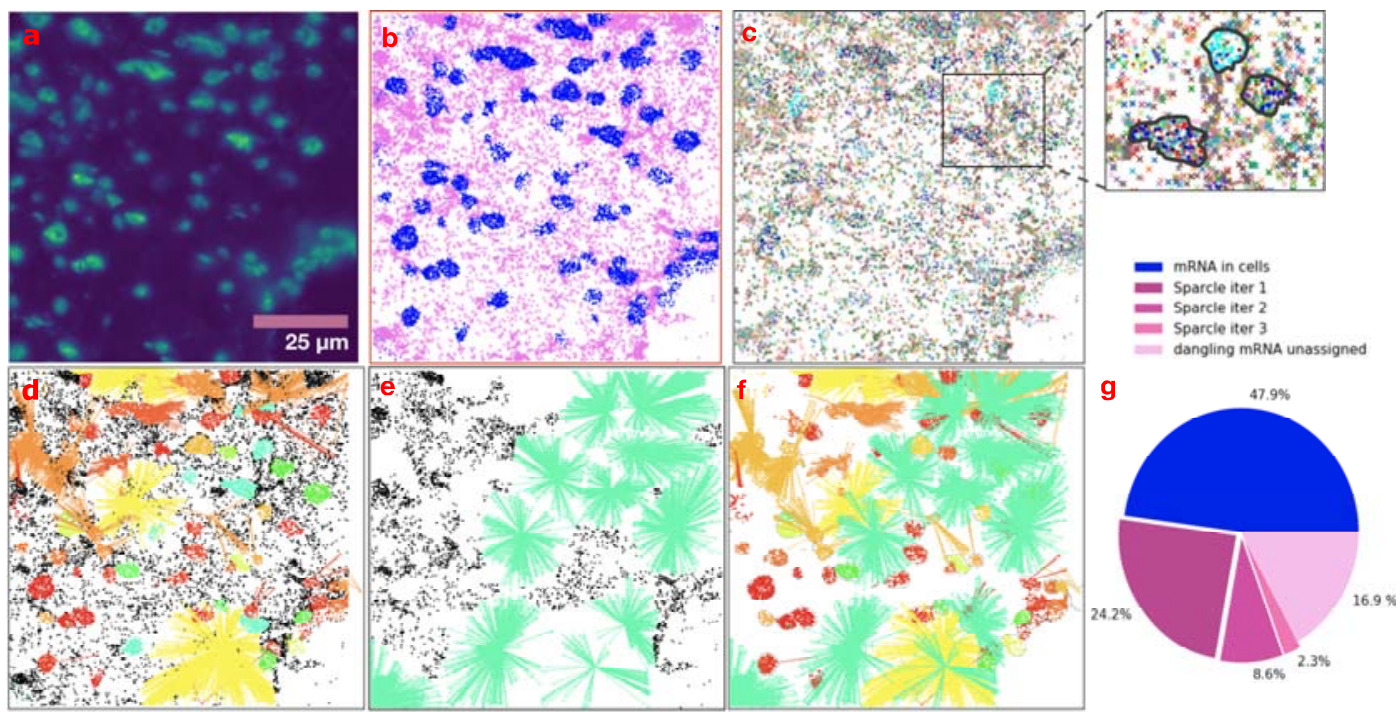

518 Figure 2: Sparcle improves cell type assignment from MERFISH data. a. (i) t-SNE

519 projections of MERFISH image data from the mouse hypothalamus optic region comprising

520400 fields of view (FOV) with $\sim 30,000$ cells total, 140 genes, with matching scRNA-seq data

521 in Moffitt et al. [9] showing diversity in cell type proportions. (ii) Percentage of cells in the

522 paired scRNA-seq data (orange bars), MERFISH (blue bars) and MERFISH with Sparcle

523 (pink bars). Post-Sparcle cell type assignment proportions are in closer accordance with the

scRNA-seq proportions, especially for astrocytes, endothelial cells, mature and immature oligodendrocytes, and excitatory and inhibitory neuronal cell types, demonstrating that

526 Sparcle can correct counts per cell type based on the dangling mRNA assignments. $\mathbf{b}$.

527 Cluster-cluster covariance (Gramian) matrices of MERFISH (left), MERFISH with Sparcle 528 (center) and scRNA-seq (right) indicate that Sparcle-treated count matrices are more similar 529 to scRNA-seq (pre-Sparcle vs. scRNA-seq median $x^{2}(2, N=\sim 61,000)=0.26, p=0.31$,

530 Frobenius norm $=15.18$; post-Sparcle vs. scRNA-seq median $\mathrm{X}^{2}(2, \mathrm{~N}=\sim 61,000)=0.95, \mathrm{p}=$

5310.62 , Frobenius norm $=2.34)$. c. Cluster-cluster cross covariance matrices between scRNA-

532 seq and MERFISH clusters pre- and post-Sparcle indicate stronger within-cluster variances

533 (dominant diagonal) after Sparcle, especially for non-neuronal cell types. d. Sparcle 534 improves the cell-type specificity of canonical gene assignment for most cell types. z-score-

535 normalized gene expression (rows) is averaged over vectors per cluster representing cell 536 type (columns). 
bioRxiv preprint doi: https://doi.org/10.1101/2021.02.13.431099; this version posted February 14, 2021. The copyright holder for this preprint (which was not certified by peer review) is the author/funder, who has granted bioRxiv a license to display the preprint in perpetuity. It is made available under aCC-BY 4.0 International license.

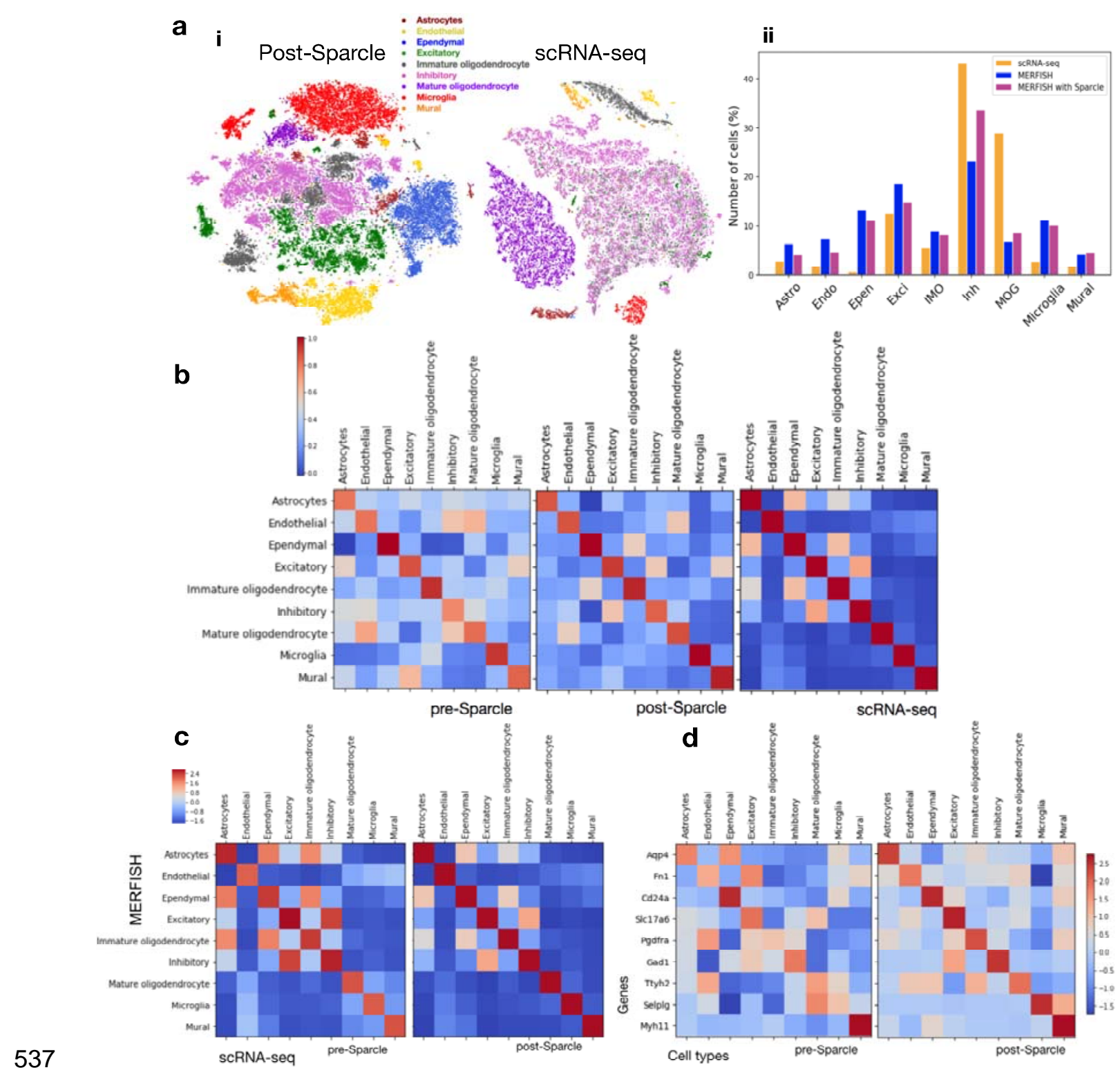

538

539

540

541

542

543 
544 Figure 3: Sparcle refines clusters in MERFISH data. a. We constructed a pre-Sparcle

545 MERFISH matrix with 100 randomly sampled excitatory cells stacked over 100 inhibitory

546 cells and a post-Sparcle matrix with the same cells, then computed Pearson correlations

547 with a similarly constructed scRNA-seq matrix of 100 random cells per cell type. Pearson

548 correlation matrices indicate greater covariance across diagonal blocks (defined red zones)

549 between scRNA-seq and post-Sparcle MERFISH data, for both excitatory and inhibitory

550 neuronal cell types. b. Similar analysis reveals the same trends for astrocytes, endothelial

551 and ependymal cells (NN set 1) and immature and mature oligodendrocytes, microglia and

552 mural cells (NN set 2).

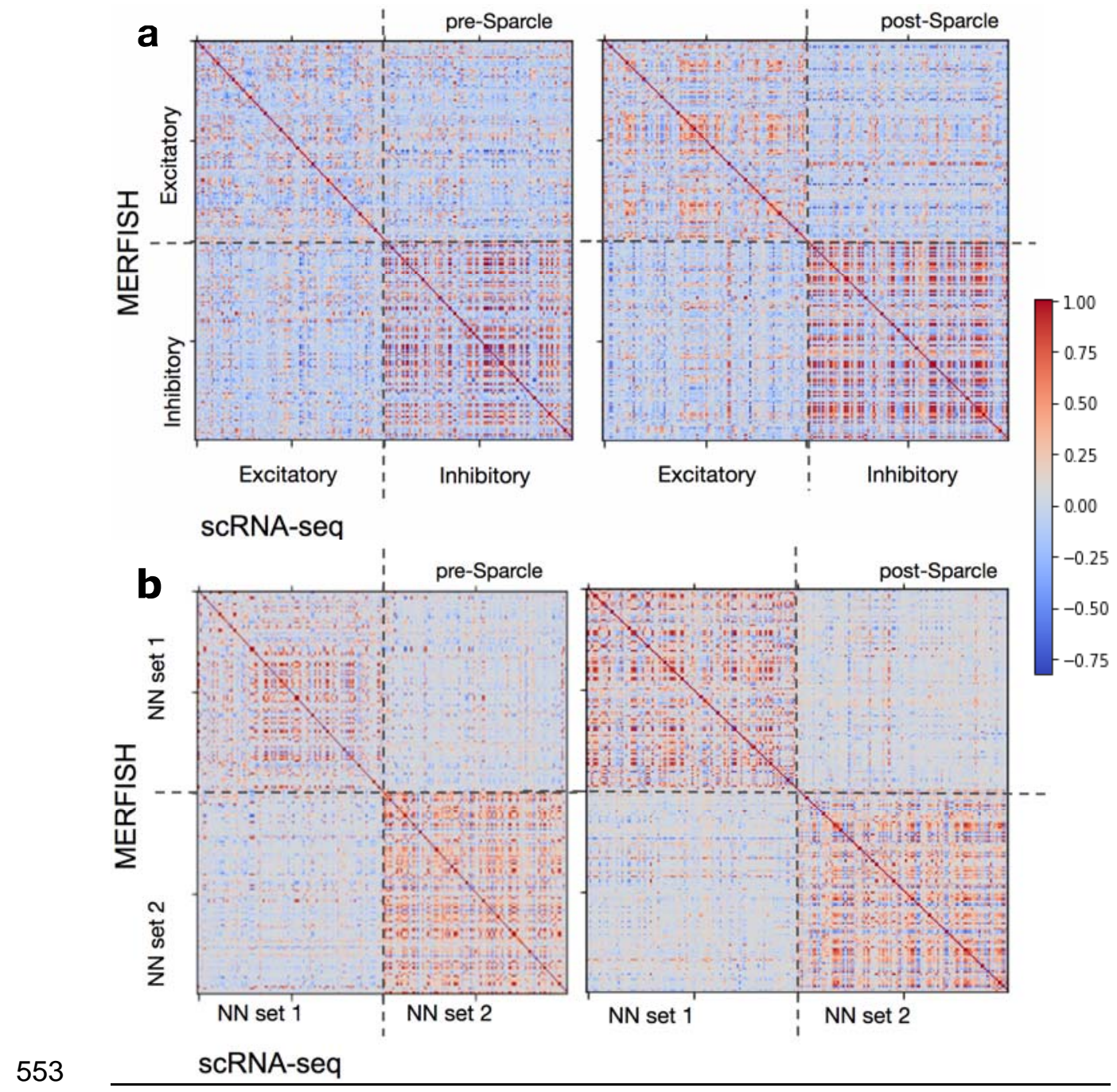


556 Figure 4: Sparcle generalizes to smFISH data. a. Single smFISH FOV of mouse visual

557 cortex containing 3500 cells and 250,000 randomly subsampled mRNAs; of 93,000 dangling

558 transcripts, 3300 are shown for visual clarity. Cells and connections to dangling mRNAs from

55922 genes are colored based on matching scRNA-seq clusters. Inset, magnification. b.

560 Cluster-cluster covariance (Gramian) matrices between (i) smFISH, (ii) smFISH with Sparcle

561 and (iii) scRNA-seq indicate that Sparcle-treated count matrices are more similar to scRNA-

562 seq (Box's $M$ test for pre-Sparcle vs. scRNA-seq median $X^{2}(2, N=3500)=0.36, p=0.48$,

563 Frobenius norm $=9.42$; Box's $M$ test for post-Sparcle vs. scRNA-seq median $X^{2}(2, N=$

$5643500)=0.92, p=0.78$, Frobenius norm $=1.83$ ). c. Cluster-cluster cross covariance matrices

565 between scRNA-seq and MERFISH clusters pre- and post-Sparcle indicate stronger within-

566 cluster variances (dominant diagonal) after Sparcle. d. Expression of 22 smFISH genes

567 (rows) within each cluster (columns) pre- and post-Sparcle, arranged by hierarchical

568 clustering of the post-Sparcle matrix. Sparcle generates clearer blocks of gene expression

569 per cluster and improves assignments for all four cell types. Color scale in all panels

570 represents z-scored values of log-normalized expression per cluster. 
bioRxiv preprint doi: https://doi.org/10.1101/2021.02 13.431099; this version posted February 14, 2021. The copyright holder for this preprint (which was not certified by peer review) is the author/funder, who has granted bioRxiv a license to display the preprint in perpetuity. It is made available under aCC-BY 4.0 International license.

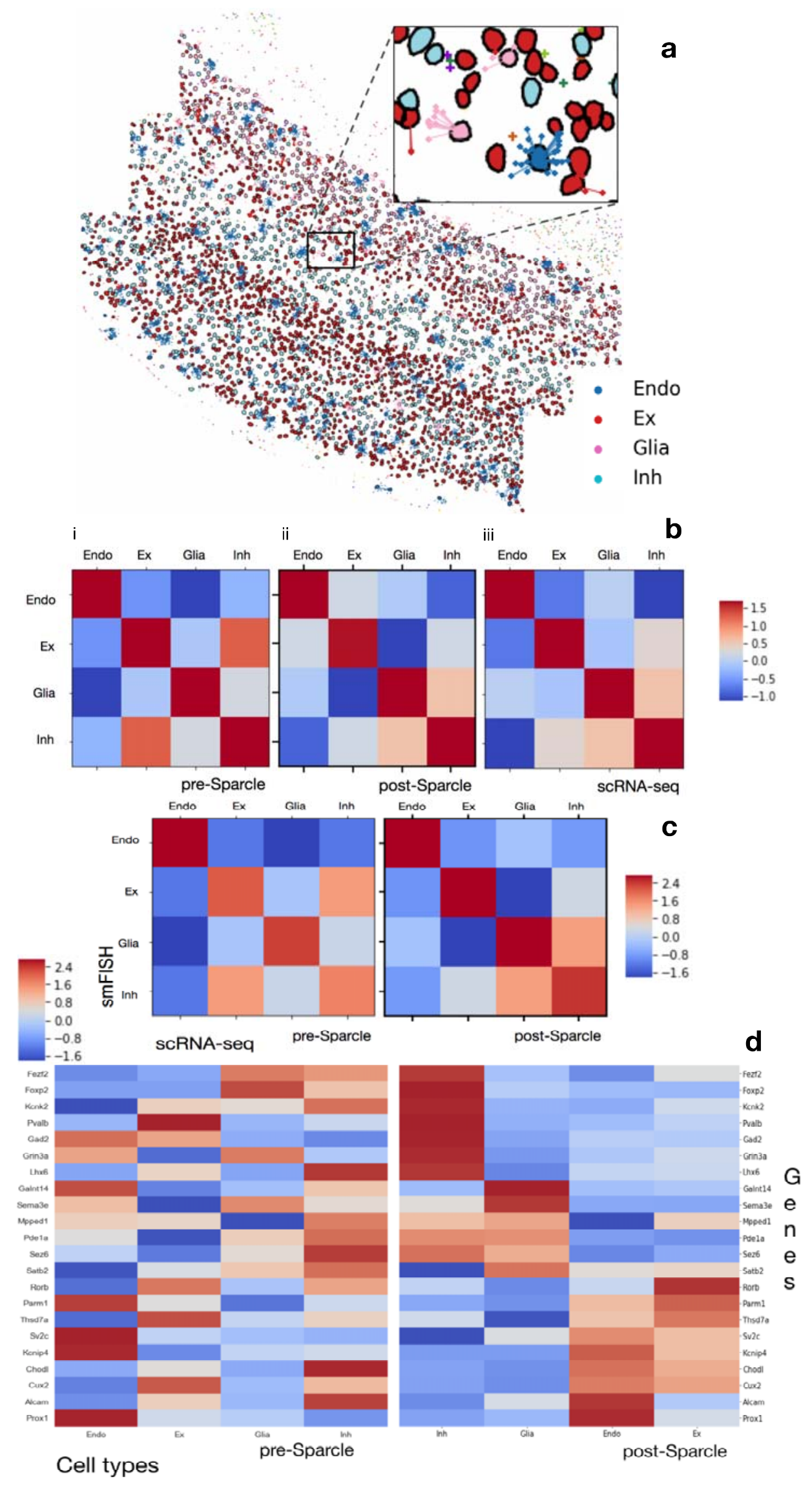


bioRxiv preprint doi: https://doi. org/10.1101/2021.02 13.431099. this version posted February 142021 . The copyright holder for this preprint (which was not certified by peer review) is the author/funder, who has granted bioRxiv a license to display the preprint in perpetuity. It is made available under aCC-BY 4.0 International license.

\section{Supplementary Figures}

573 Figure S1: Weighted Mockcell creation. A MERFISH FOV showing spatial allocation of 134

574 genes in a section of the mouse hypothalamus optic region [9]. Dots represent mRNA within

575 cell segmentations, colored by cell cluster assignment. Crosses represent unassigned

576 (dangling) mRNA. Inset, a zoomed-in region showing mock cell (blue circle), centred at a

577 dangling mRNA (pink cross). All mRNA (dots and crosses) within the circle will contribute to

578 that mock cell, weighted inversely to the distance from the central mRNA.

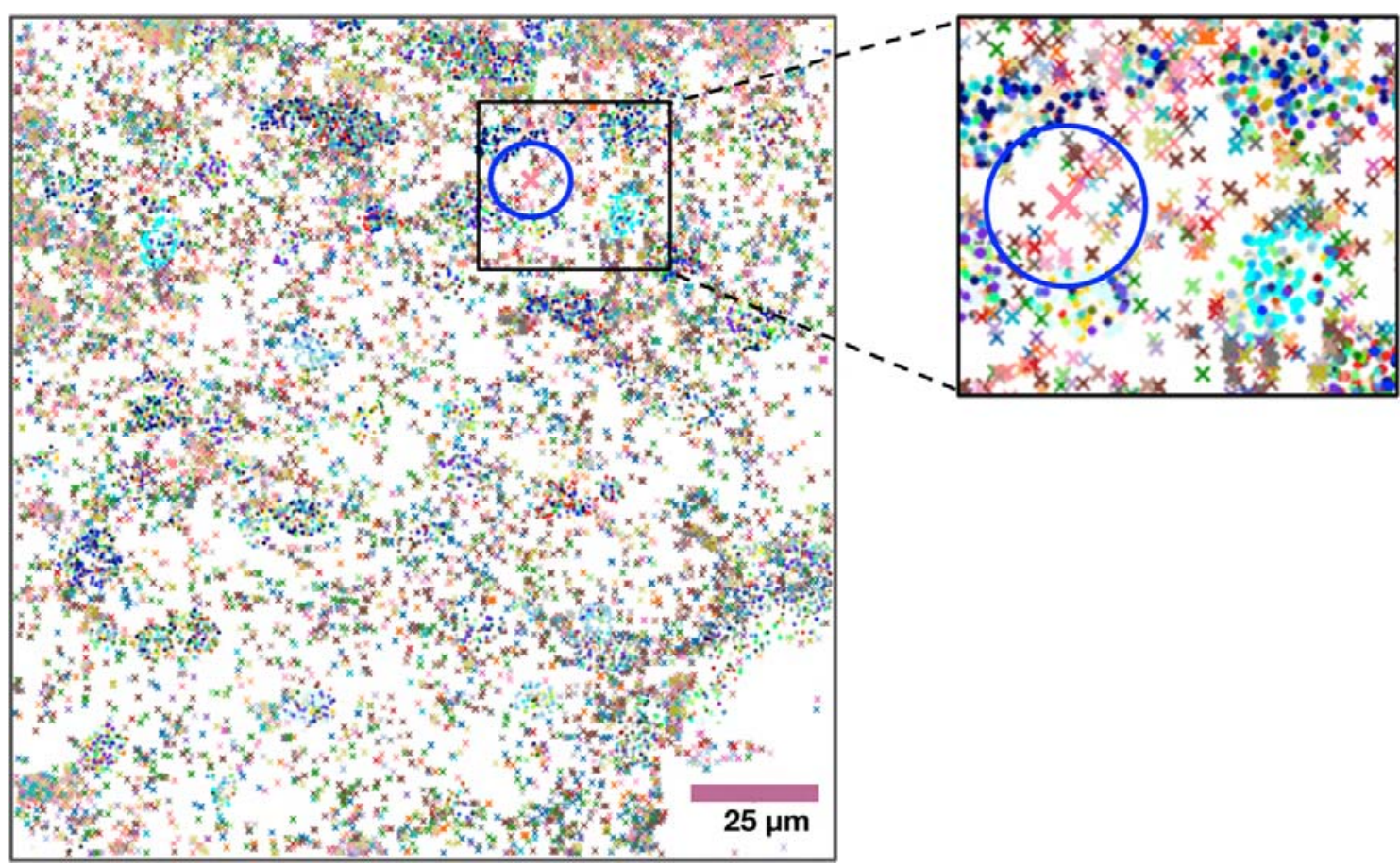

580

581

582

583

584

585

586

587 
588 Figure S2: Mockcell creation. a. A MERFISH image shown in image coordinates after

589 perspective projection from stage coordinates. This projection allows the $\mathrm{x}$ and $\mathrm{y}$ axis to start 590 from $(0,0)$ and enables the calculation of Euclidean distances between cell centroids and 591 mRNA transcripts. We use this to estimate the radius $r$ for a mockcell. For example, we draw

592 a bounding box around cell $A$ and cell $B$ and observe that its radius is around 80 pixels. This 593 is used as a proxy for the mockcell creation. b. The same MERFISH image recreated in 594 Python using cell segmentation coordinates to outline the neuronal cells. The cells are 595 colored based on their cell type. A dangling mRNA is shown as a black cross with red 596 crosses, traced within a circle of radius 80 pixels, identifying those mRNA that will contribute 597 to its mockcell.

598
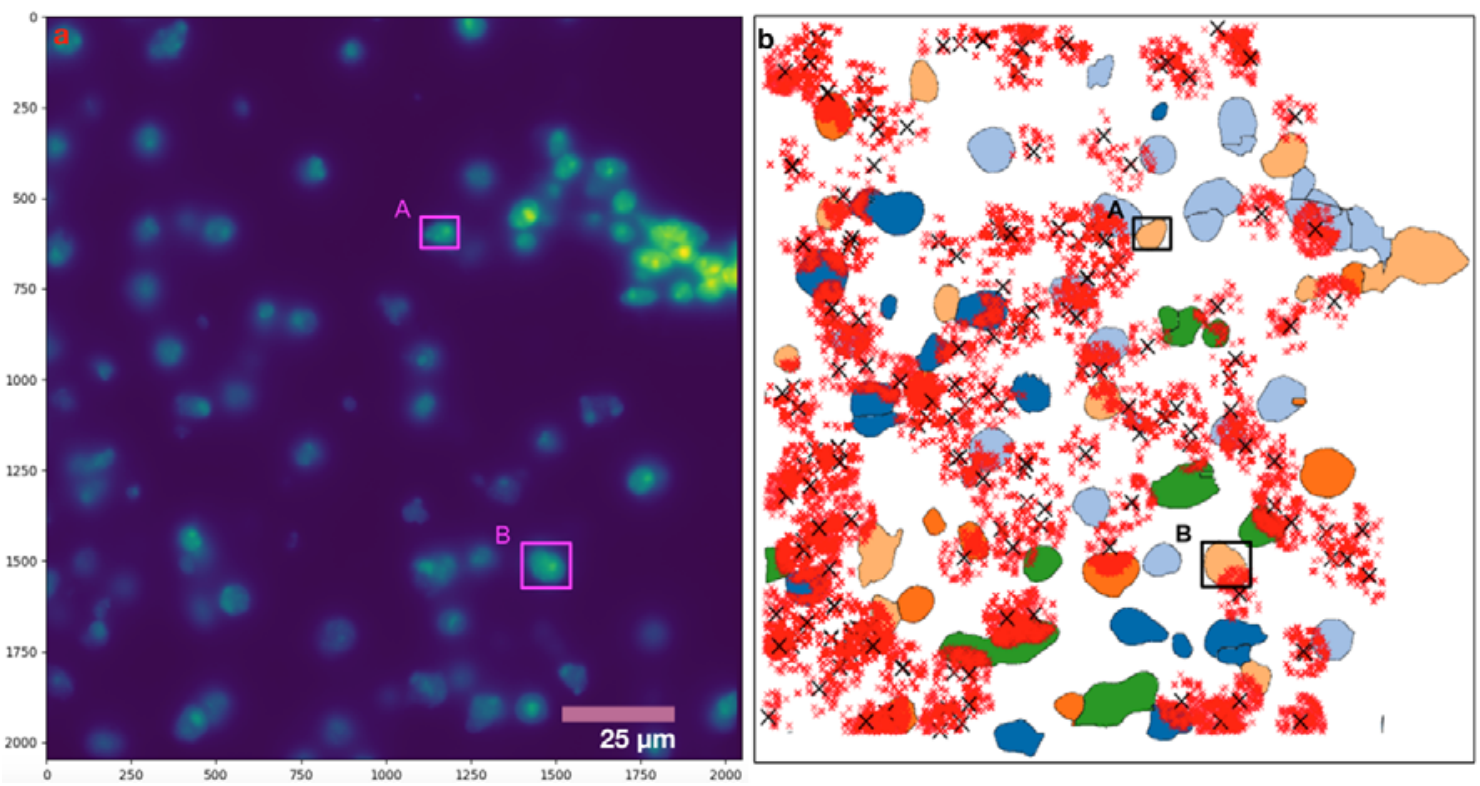
bioRxiv preprint doi: https://doi org/10.1101/2021.02.13.431099. this version posted February 14,2021 . The copyright holder for this preprint (which was not certified by peer review) is the author/funder, who has granted bioRxiv a license to display the preprint in perpetuity. It is made available under aCC-BY 4.0 International license.

607

608 Figure S3: Allen smFISH data and matching scRNA-seq data. a. Single smFISH FOV of 609 mouse visual cortex containing 3500 cells and 250,000 randomly subsampled mRNAs; of 61093,000 dangling transcripts, 3300 are shown for visual clarity. Cells and dangling mRNAs 611 are colored based on matching scRNA-seq clusters. Inset, magnification with dangling 612 mRNAs (+ symbols). The dangling mRNAs account for $22 \mathrm{smFISH}$ genes: Alcam, Chodl, 613 Cux2, Fezf2, Foxp2, Gad2, Galnt14, Grin3a, Kcnip4, Kcnk2, Lhx6, Mpped1, Parm1, Pde1a, 614 Prox1, Pvalb, Rorb, Satb2, Sema3e, Sez6, Sv2c, Thsd7a. b. tSNE projection showing 615 proportions of the four hierarchical clusters corresponding to endothelial (Endo), excitatory 616 (Ex), glial (Glia) and inhibitory (Inh) cell types of the matching scRNA-seq data, which 617 comprises 43,498 cells and

61845,000 genes.
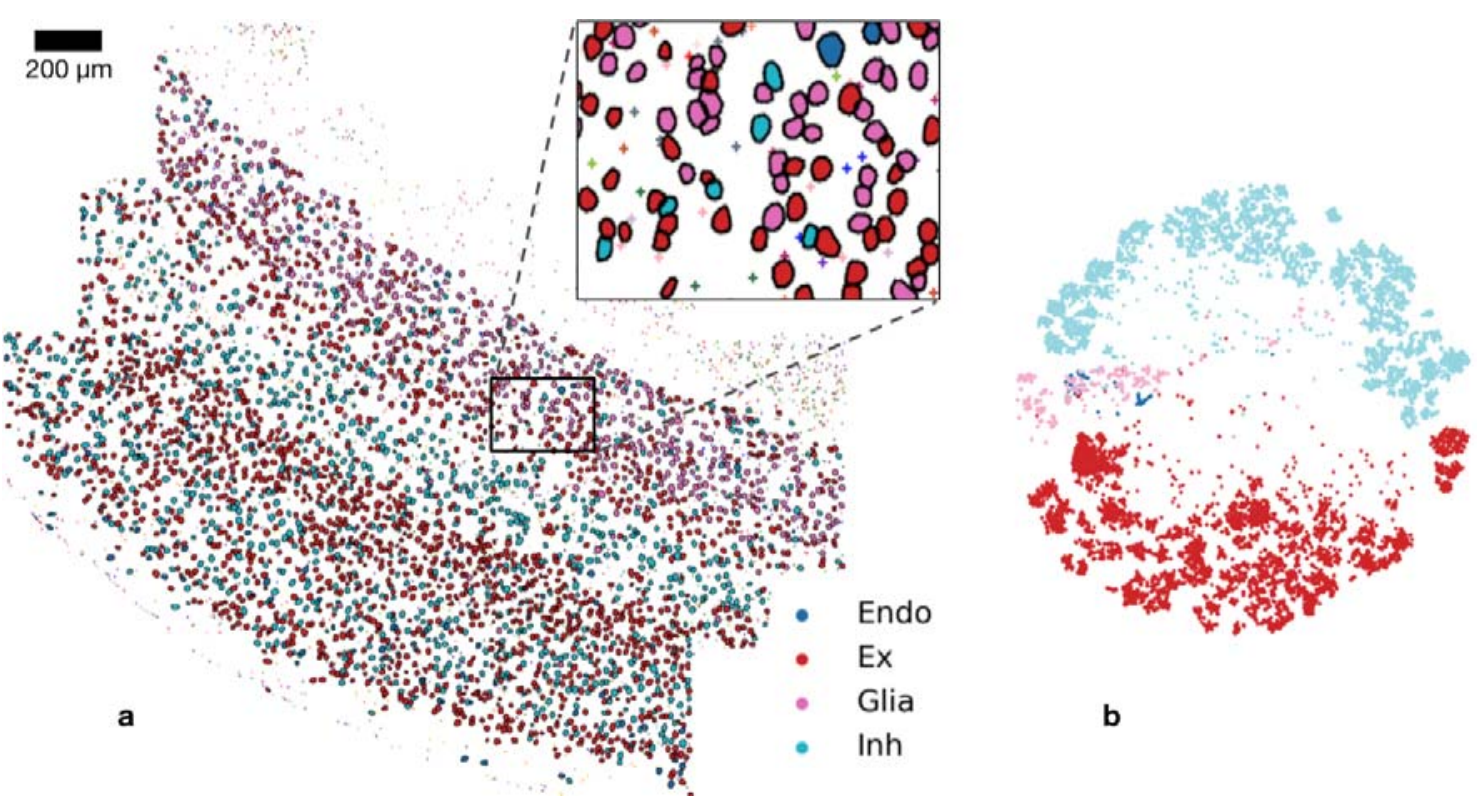

620

621

622

623

624

625 
628 Figure S4: Percentage of mRNA recovery across the 4 dominant cell types using Sparcle in 629 the Allen smFISH data. We subsample 250,000 mRNAs from a total of 1,074,000 within this 630 image; 154,000 mRNAs (61.8\%) are within cells and 95,000 (38.2\%) are dangling. Cell type 631 proportions of within-cell mRNA in the smFISH data are in blue (endothelial $8.1 \%$, excitatory $63222.7 \%$, glial 11.8\%, inhibitory 19.3\%), and additional dangling transcripts assigned by 633 Sparcle are in pink, comprising $32.2 \%$ of the total mRNA (endothelial $5.5 \%$, excitatory $63411.25 \%$, glial $6.1 \%$, inhibitory $9.3 \%$ ) across three iterations; only $6 \%$ of the total mRNA 635 remains unassigned.

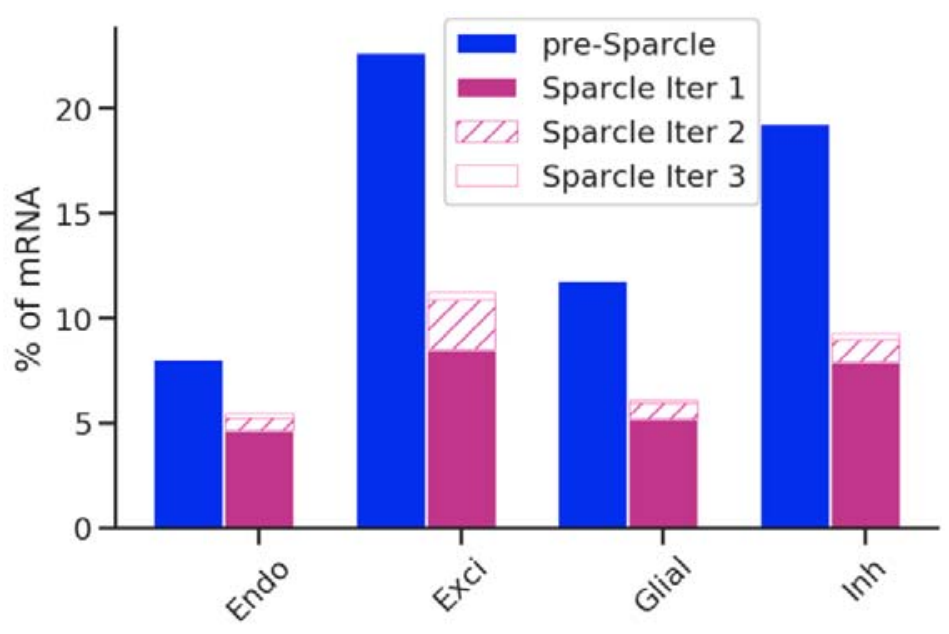




\section{References}

1. Chen $\mathrm{KH}$, Boettiger AN, Moffitt JR, Wang S, Zhuang X. RNA imaging. Spatially resolved, highly multiplexed RNA profiling in single cells. Science. 2015;348(6233).

2. Codeluppi S, Borm L, Zeisel A, La Manno G, van Lunteren J, Svensson C, et al. Spatial organization of the somatosensory cortex revealed by osmFISH. Nature Methods. 2018;15(11):932-5.

3. Eng CL, Lawson M, Zhu Q, Dries R, Koulena N, Takei Y, et al. Transcriptome-scale super-resolved imaging in tissues by RNA seqFISH. Nature. 2019;568(7751):235-9.

4. Long X, Colonell J, Wong A, Singer R, Lionnet T. Quantitative mRNA imaging throughout the entire Drosophila brain. Nature Methods. 2017;14(7):703-6.

5. Lubeck E, Coskun A, Zhiyentayev T, Ahmad M, Cai L. Single-cell in situ RNA profiling by sequential hybridization. Nature Methods. 2014;11(4):360-1.

6. Nichterwitz S, Chen G, Aguila BJ, Yilmaz M, Storvall H, Cao M, et al. Laser capture microscopy coupled with Smart-seq2 for precise spatial transcriptomic profiling. Nature Communications. 2016;7(12139).

7. Battich N, Stoeger T, Pelkmans L. Image-based transcriptomics in thousands of single human cells at single-molecule resolution. Nature Methods. 2013;10(11):1127-33.

8. Shah S, Lubeck E, Zhou W, Cai L. In situ transcription profiling of single cells reveals spatial organization of cells in the mouse hippocampus. Neuron. 2016;92(2):342-57.

9. Moffitt J, Bambah-Mukku D, Eichhorn S, Vaughn E, Shekhar K, Perez J, et al. Molecular, spatial, and functional single-cell profiling of the hypothalamic preoptic region. Science. 2018;362(6416).

10. Braiki M, Benzinou A, Nasreddine K, Hymery N. Automatic Human Dendritic Cells Segmentation Using K-Means Clustering and Chan-Vese Active Contour Model. Comput Methods Programs Biomed. 2020;195(105520).

11. Neal RM. Markov chain sampling methods for Dirichlet process mixture models. Journal of Computational and Graphical Statistics. 2000;9:249-65.

12. Teh YW, Jordan MI, Beal MJ, Blei DM. Hierarchical dirichlet processes. Journal of the American Statistical Association. 2006;101(476):1556-81.

13. Prabhakaran S, Azizi E, Carr A, Pe'er D. Dirichlet Process Mixture Model for Correcting Technical Variation in Single-Cell Gene Expression Data. Journal of Machine Learning Research (JMLR) Workshop and Conference Proceedings. 2016;48:1070-9. 14. Levine J, Simonds E, Bendall S, Davis K, Amir e-A, Tadmor M, et al. Data-Driven Phenotypic Dissection of AML Reveals Progenitor-like Cells that Correlate with Prognosis. Cell. 2015;162(1):184-97.

15. Allen Brain Map. Cell Types Database: RNA-Seq Data https://portal.brainmap.org/atlases-and-data/rnaseq\#Mouse Cortex and Hip [

16. Park J, Choi W, Tiesmeyer S, Long B, Borm LE, Garren E, et al. Segmentation-free inference of cell types from in situ transcriptomics data. biorXiv. 2019.

17. Petukhov V, Soldatov RA, Khodosevich K, Kharchenko PV. Bayesian segmentation of spatially resolved transcriptomics data. biorXiv. 2020.

18. Shepard D. A two-dimensional interpolation function for irregularly-spaced data. ACM National Conference. 1968:517-24.

19. Myung IJ. Tutorial on maximum likelihood estimation. Journal of mathematical Psychology. 2003;47(1):90-100.

20. Bindel D, Demmel J, Kahan W, Marques O. On Computing Givens rotations reliably and efficiently. LAPACK Working Note 148. 2001. 
bioRxiv preprint doi: https://doi.org/10.1101/2021.02 13.431099; this version posted February 14, 2021. The copyright holder for this preprint

(which was not certified by peer review) is the author/funder, who has granted bioRxiv a license to display the preprint in perpetuity. It is made available under aCC-BY 4.0 International license.

704 21. Bartel D. qr_decomposition 2015. Available from:

705 https://github.com/danbar/ar decomposition.

706 22. Box GEP. A general distribution theory for a class of likelihood criteria. Biometrika.

707 1949;36:317-46

708 23. Hahs-Vaughn D. Applied Multivariate Statistical Concepts: Taylor \& Francis; 2016.

709 24. Glen S. Box's M Test: Definition [Available from:

710 https://www.statisticshowto.com/boxs-m-test/.

711 25. Rao CR. Linear Statistical Inference and its Applications. 2 ed: Wiley; 1973. 